\title{
Selective Breeding, Quantitative Trait Locus Analysis, and Gene Arrays Identify Candidate Genes for Complex Drug- Related Behaviors
}

\author{
Boris Tabakoff, Sanjiv V. Bhave, and Paula L. Hoffman \\ Department of Pharmacology, University of Colorado Health Sciences Center, Denver, Colorado 80262
}

\begin{abstract}
Acute functional tolerance to ethanol develops during a single exposure to ethanol; it has been suggested to be a predisposing factor for the development of ethanol dependence. Genetic determinants of acute functional tolerance, as well as of ethanol dependence, have been clearly demonstrated. We describe a novel approach that uses a combination of selective breeding (to segregate genes contributing to the phenotype of interest, i.e., acute functional tolerance to the incoordinating effect of ethanol), quantitative trait locus analysis (to define chromosomal regions associated with acute functional tolerance), and DNA microarray technology (to identify differentially expressed genes in the brains of the selected lines of mice) to identify candidate genes for the complex phenotype of ethanol tolerance. The results indicate the importance of a signal transduction cascade that involves the glutamate receptor $\delta 2$ protein, the Ephrin B3 ligand, and the NMDA receptor, as well as a transcriptional regulatory protein that may be induced by activation of the NMDA receptor (zinc finger protein 179) and a protein that can modulate downstream responses to NMDA receptor activation (peroxiredoxin), in mediating acute tolerance to the incoordinating effect of ethanol.
\end{abstract}

Key words: QTL analysis; DNA microarrays; selective breeding; acute functional ethanol tolerance; candidate genes; mice

\section{Introduction}

Tolerance to beverage alcohol (ethanol) is an important component of the Diagnostic and Statistical Manual of Mental Disorders (DSM-IV) and International Classification of Diseases (ICD-10) diagnoses of alcohol dependence (World Health Organization, 1993; American Psychiatric Association, 1994). Tolerance that develops during a single exposure to alcohol is defined as acute functional tolerance (AFT), or within-session tolerance, and has been considered a predisposing element for alcohol dependence (Newlin and Thomson, 1990). A genetic component to AFT to alcohol has clearly been demonstrated by the bidirectional selective breeding of lines of mice to display high acute functional tolerance (HAFT mice) and low acute functional tolerance (LAFT mice) to the incoordinating effect of ethanol (Erwin and Deitrich, 1996). The technique of selective breeding enhances the representation (frequency) of genetic material associated with a particular trait, which shifts the animal's phenotype from the population mean (Falconer and Mackay, 1996). Therefore, the expectation is that HAFT mice have more genetic material that promotes the higher level of AFT, whereas the LAFT animals accumulate less of this genetic material and/or more genetic material leading to lower AFT (Falconer and Mackay, 1996).

The chromosomal location of particular genes that may influence AFT to ethanol has been investigated recently with quantitative trait locus (QTL) analysis (Kirstein et al., 2002). This tech-

\footnotetext{
Received Jan. 27, 2003; revised March 14, 2003; accepted March 18, 2003.

This work was supported in part by the National Institute on Alcohol Abuse and Alcoholism and the Banbury Fund. We thank Drs. Razvan Lapadat, Tzu Phang, Sonia Leach, Imran Shah, and Lawrence Hunter of the Center for Computational Pharmacology, University of Colorado Health Sciences Center for their assistance.

Correspondence should be addressed to Boris Tabakoff, Department of Pharmacology C-236, University of Colorado Health Sciences Center, 4200 East Ninth Avenue, Denver, C0 80262. E-mail: boris.tabakoff@uchsc.edu. Copyright $\odot 2003$ Society for Neuroscience $\quad$ 0270-6474/03/234491-08\$15.00/0
}

nique is a statistical analysis of the association between a complex phenotype (i.e., the degree of acute tolerance expressed in individual animals) and the occurrence of specific marker alleles in the animal's genome (Crabbe et al., 1999). However, QTL analysis does not identify the critical gene or genes within relatively large chromosomal regions defined by the QTL. The genetic difference underlying the QTL could be a polymorphism in the coding region of the gene, leading to a difference in the functional activity of the gene product, a polymorphism that could alter the level of transcript produced or the stability of the transcript, or all of these factors.

In the present study, we have used DNA microarray analysis to identify genes that are differentially expressed in the brains of HAFT and LAFT mice. These gene expression experiments would be expected to provide an indication of the genetic elements that contribute to the phenotypic differences between the selected lines through the level of transcript being produced. However, without additional information, it is difficult or impossible to ascribe a definitive role to any particular differentially expressed gene in contributing to AFT (Crabbe et al., 1990; Falconer and Mackay, 1996). On the other hand, if the differentially expressed genes in selectively bred animals are located within a QTL for the same phenotypic trait for which the animals are bred, more confidence can be allotted to the possibility that a differentially expressed gene is a predisposing factor for the expression of the selected phenotype. Therefore, we also mapped the genes that were differentially expressed in the brains of HAFT and LAFT mice to the identified QTL regions for AFT, resulting in a set of genes that may be considered to contribute to this trait. We gained significant power in this analysis from the availability of replicate selected mouse lines for AFT (Crabbe et al., 1999).

There have been a limited number of other reports that have 
Table 1. Analysis of RNA from replicate lines of HAFT and LAFT mice on Affymetrix oligonucleotide arrays

\begin{tabular}{|c|c|c|c|}
\hline & MAS version 4.0 & MAS version 5.0 & Common probes \\
\hline Total transcripts analyzed & 4305 & 3989 & 3884 \\
\hline Analysis mode & \multicolumn{2}{|c|}{$\begin{array}{l}\text { Number of transcripts with } t \text { value exceeding the percentile of the } \\
\text { control distribution }{ }^{a}\end{array}$} & \\
\hline \multicolumn{4}{|c|}{$t$ Test noise distribution percentile } \\
\hline 95 & 33 & 26 & 9 \\
\hline 90 & 105 & 101 & 35 \\
\hline 85 & 231 & 207 & 88 \\
\hline 80 & 371 & 395 & 177 \\
\hline \multicolumn{4}{|l|}{ Permutation ${ }^{b}$ percentile } \\
\hline 95 & 75 & 71 & 22 \\
\hline 90 & 189 & 166 & 63 \\
\hline 85 & 287 & 269 & 115 \\
\hline 80 & 384 & 353 & 169 \\
\hline
\end{tabular}

${ }^{a}$ Differential expression in same direction in both replicate lines.

${ }^{b} 100$ permutations.

Total RNA from whole brain of five individual HAFT-1 or LAFT-1 mice, or four individual HAFT-2 or LAFT-2 mice, was used to generate cRNA, which was hybridized to individual Affymetrix MGU74A oligonucleotide arrays. Array data were normalized by global scaling, and present and absent calls were determined using Affymetrix MAS version 4.0 or version 5.0 . Transcripts that were present on all arrays, or present on all arrays for one line and absent/marginal/present on all arrays from the other line, were used for analysis. "Total transcripts analyzed" are those that overlapped between the two replicate lines. "Common probes" represent the transcripts that overlapped between MAS version 4.0 and MAS version 5.0. The expected percentage of false positive values is as follows: 95 th percentile, $0.5 \% ; 90$ th percentile, $2.0 \% ; 85$ th percentile, $4.5 \% ; 80$ th percentile, $8.0 \%$ of the total number of transcripts analyzed (see Materials and Methods).

combined DNA microarray analysis with QTL results to assess genes that contribute to allergen-induced airway hyperresponsiveness (Karp et al., 2000), hypertension (Aitman et al., 1999), or diabetes (Eaves et al., 2002). However, the present study is the first to examine the genetic underpinning of a behavioral phenotype using a combination of selective breeding, QTL analysis, and gene expression analysis.

\section{Materials and Methods}

Mice

Replicate selected lines of HAFT and LAFT mice (HAFT-1/LAFT-1, HAFT-2/LAFT-2) were generated as described previously (Erwin and Deitrich, 1996) and were obtained from the Institute for Behavioral Genetics, Boulder, CO. Male mice, $\sim 10$ weeks old, were used for these experiments.

\section{Measurement of AFT to ethanol}

The measurement of AFT to ethanol was performed in mice of generation 22 of the HAFT-1, LAFT-1 selection and generation 20 of the replicate HAFT-2, LAFT-2 selection as described previously (Erwin and Deitrich, 1996; Kirstein et al., 2002). Mice were trained to balance on a stationary dowel and were then given an injection of $1.75 \mathrm{gm} / \mathrm{kg}$ of ethanol, i.p. At the time that a mouse lost balance on the dowel a retroorbital sinus blood sample was obtained for measurement of the blood ethanol concentration (BEC0). A second blood sample was obtained when the mouse regained balance on the dowel (BEC1). At this time, a second ethanol injection was given $(2.0 \mathrm{gm} / \mathrm{kg}$, i.p.); a third blood sample was taken when the mouse again regained balance on the dowel (BEC2). Blood ethanol concentrations were determined by gas chromatography, and the difference between BEC2 and BEC1 was the measure of AFT. In the QTL analysis (Kirstein et al., 2002), AFT and BEC2 were found to be significantly and positively genetically correlated $(r=0.78)$ measures, whereas AFT and BEC1 $(r=0.23)$ were not significantly genetically correlated.

The QTLs reported by Kirstein et al. (2002) are considered to be provisional. There were eight total QTLs reported for AFT and/or BEC2, of which three attained $p<0.002$ or better ("suggestive" by the criteria of Lander and Kruglyak, 1995). At the $p<0.002$ level or better, on average, there would be one false positive expected in a total genome scan ( 1 of 3 , or 33\%, false positives). The other five QTLs reported for AFT and/or BEC2 ranged from $p<0.004$ to $p<0.009$ (Kirstein et al., 2002). These QTLs can be considered more likely to be true than false positives, although the expected false positive rate would be higher than 33\% (i.e., between 33 and 50\%) (Belknap et al., 1996; John Belknap, Oregon Health Sciences University, Portland, OR, personal communication). Our six definitively identified genes are localized within two QTLs attaining $p<$ 0.002 or better, and within two QTLs at the level of $p<0.006$.

To confirm line identification, HAFT and LAFT mice were genotyped using a panel of microsatellite markers (Prism Mouse Mapping Primers version 1.0; Applied Biosystems International, Foster City, CA). Tail (25-50 mg tissue) DNA was isolated using the DNeasy kit (Qiagen, Valencia, CA), and genotyping was performed using the Applied Biosystems International Prism 7000 Sequence Detection System.

\section{Gene expression analysis}

Total RNA extraction. Naive HAFT and LAFT mice were killed by $\mathrm{CO}_{2}$ exposure, and whole brains were removed and frozen on dry ice. Brains were stored at $-70^{\circ} \mathrm{C}$ until used. Total RNA was extracted from whole brains using the TRIzol reagent (Invitrogen, Carlsbad, CA). An additional clean-up of total RNA was performed using the RNeasy kit (Qiagen).

cRNA preparation for GeneChip expression analysis. Affymetrix GeneChip oligonucleotide arrays (MGU74A arrays versions 1.0 and 2.0; Affymetrix, Santa Clara, CA) were used in these experiments. The mask file provided by Affymetrix (Mg_U74a.msk) was used to mask defective probe sets on the MGU74A version 1 array. Using the protocol supplied by the manufacturer, double-stranded cDNA was synthesized from total RNA and was used to obtain biotin-labeled cRNA by an in vitro transcription reaction. Biotin-labeled cRNA was fragmented and hybridized with the GeneChip Arrays, according to the manufacturer's protocol, after verifying the quality of the biotin-labeled cRNA on a TestChip. The array was stained with streptavidin-phycoerythrin conjugate and scanned by an Affymetrix GeneArray scanner. RNA from five individual HAFT-1, five LAFT-1, four HAFT-2, and four LAFT-2 mice was individually hybridized to 18 microarrays. RNA from HAFT-1 and LAFT-1 mice was hybridized to the MGU74A version 1 array, and RNA from HAFT-2 and LAFT-2 mice was hybridized to the MGU74A version 2 arrays. We later determined that transcripts present on one version of the array, but either not present or masked on the other, were not localized within the relevant QTL regions.

Data analysis. The image data obtained from the Affymetrix GeneChip arrays were initially analyzed with two different programs provided by Affymetrix. With each program, a background value is subtracted and a noise correction is applied. A global scaling procedure was used for chipto-chip normalization. Microarray Suite (MAS) version 4.0 then uses an empirical algorithm to determine "present" and "absent" calls and fluorescence intensity levels (average difference values), whereas MAS version 5.0 uses a statistical algorithm to determine the presence or absence and "signal" intensity (Affymetrix 2001). MAS version 5.0 includes a detection algorithm that uses probe pair (perfect match/mismatch) in- 
Table 2. Differentially expressed genes in brains of HAFT and LAFT mice ${ }^{a}$

\begin{tabular}{|c|c|c|c|c|c|c|}
\hline $\begin{array}{l}\text { Gene } \\
\text { name }\end{array}$ & Description & $\begin{array}{l}\text { HAFT-1/ } \\
\text { LAFT-1 (\%) }\end{array}$ & $\begin{array}{l}\text { HAFT-2/ } \\
\text { LAFT-2 (\%) }\end{array}$ & $\begin{array}{l}\text { Chromosomal localization } \\
\text { (chromosome: cM) }\end{array}$ & $\begin{array}{l}\text { QTL/marker } \\
\text { location }^{b}\end{array}$ & QTL $p^{b}$ \\
\hline Grid2 & GluR $\delta 2$ receptor & 139 & 136 & $6: 29.65$ & $6: 30.5-35.8$ & 0.006 \\
\hline Efnb3 & Ephrin B3 ligand & 119 & 116 & $11: 40$ & $11: 29-43$ & 0.002 \\
\hline Grin1 & NMDAR1 & 190 & 154 & 2:12 & 2:11 & 0.006 \\
\hline Prdx5 & Peroxiredoxin 5 & 87 & 92 & 1:83.6 & $1: 81.1-89.2$ & 0.001 \\
\hline Zfp179 & Zinc finger protein 179 & 159 & 171 & $11: 34.5$ & $11: 29-43$ & 0.002 \\
\hline Tceb11 & Transcription elongation factor & 137 & 127 & 11:31 & $11: 29-43$ & 0.002 \\
\hline Grial $^{c}$ & GluR1 (AMPA receptor) & 122 & 138 & $11: 31$ & $11: 29-43$ & 0.002 \\
\hline $\operatorname{Sec} 8^{d}$ & S. cerevisiae homolog & 120 & 162 & $6: 12$ & $6: 10-21$ & 0.001 \\
\hline $\operatorname{Rad} 50^{d}$ & S. cerevisiae homolog & 78 & 89 & $11: 28.9$ & $11: 29-43$ & 0.002 \\
\hline Catna $^{d}$ & catenin $\alpha_{2}$ & 85 & 82 & $6: 34.2$ & $6: 30.5-35.8$ & 0.006 \\
\hline $\mathrm{B} 2 \mathrm{~m}^{d}$ & $\beta 2$ microglobulin & 80 & 75 & $2: 69$ & $2: 69-71$ & 0.005 \\
\hline
\end{tabular}

${ }^{a}$ All of the genes were identified as being differentially expressed regardless of the statistical method used. The genes indicated in bold were also found to be differentially expressed regardless of whether MAS version 4.0 or MAS version 5.0 was used. The combination of permutation and MAS version 4.0 also identified solute carrier family 30 (zinc transporter), member 4, as being differentially expressed (chromosomal localization, 2:69; QTL 2:69-71). However, this transcript was not identified by any other combination of analyses.

${ }^{b} \mathrm{QTL}$ data are from Kirstein et al. (2002) and represent chromosome: CM. These QTLs are regarded as provisional. Genes were considered to be within the QTL if their chromosomal location was within the range of the QTL, or was within 1 CM of the marker used to define the QTL. Results of the two-way ANOVA: effect of line: MAS version 4.0: Grid2, $F=10.6, p<0.006 ; E f n b 3, F=8.4, p<0.01 ; G r i n 1, F=9.8, p<0.007 ;$ Prdx5, $F=6.0, p<0.03 ; Z f f p 179, F=19.1, p<$ $0.001 ;$ Tceb11, $F=20.2, p<0.001$; effect of line, MAS version 5.0: Grid2, $F=16.7, p<0.001 ; E f n b 3, F=13.7, p<0.002 ; G r i n 1, F=18.8, p<0.001 ; P r d x 5, F=10.0, p<0.007 ; Z f p 179, F=24.6, p<0.001 ;$ Tceb11, $F=25.3, p<$ 0.001 .

'This gene was identified as being differentially expressed only when data were initially analyzed with MAS version 4.0 .

${ }^{d}$ These genes were identified as being differentially expressed only when data were initially analyzed with MAS version 5.0 .

tensities to generate a detection $p$ value that is used to determine whether a transcript is present or absent. For our analysis, the default threshold for a present call was used. Although MAS version 4.0 analysis can result in negative average difference calls (meaning that hybridization to mismatch probes is higher than hybridization to perfect match probes), the algorithm used for MAS version 5.0 eliminates this phenomenon. Because of the different algorithms used by MAS version 4.0 and MAS version 5.0, different numbers of transcripts can be called present or absent by the two programs. Gene lists can be found in the supplementary information posted at http://www2.uchsc.edu/pharm/faculty/tabakoff/suppl.asp.

In each case, transcripts that were present on all microarrays in a given experiment (e.g., all five HAFT-1 and all five LAFT-1), or were present on all chips from one line (e.g., HAFT-1) and absent/marginal/present on all chips in the other line (e.g., LAFT-1) were subjected to additional analysis to determine differential expression. Average difference or signal values were subjected to log transformation (negative average difference values in MAS version 4.0 were assigned a value of 1 ), and two types of statistical analysis were performed to identify genes that were differentially expressed between HAFT and LAFT mice. The first is similar to that described by Eaves et al. (2002). Differences in gene expression were assessed by calculating $t$ statistics for each measurement. A filter was then applied that required a minimum threshold value for $t$. Those thresholds were estimated based on $t$ values for genes with positive values for $t$ in one replicate group (e.g., HAFT-1 vs LAFT-1) and negative values for $t$ in the other replicate group (HAFT-2 vs LAFT-2). That is, transcripts that were upregulated in one replicate group and downregulated in the other replicate group were used to establish the control distribution of $t$ values for each replicate set, independently of any "real" changes. We then required that the value of $t$ for each comparison exceed a given percentile for this control distribution (Table 1). At each percentile level for the control distribution, the expected number of false positives could be calculated: $(1-$ percentile) [error rate experiment 1 ] $\times(1-$ percentile $)$ [error rate experiment 2] $\times$ (number of genes $) \times 2$ [each direction] $=$ number of expected false positives per array-wide comparison (Eaves et al., 2002). This method was chosen because it allowed optimal use of the whole dataset derived from the replicate selected lines of mice, and should be generally applicable to studies in which such replicate lines are available.

The second statistical analysis used was a permutation procedure, in which the group identities of the samples are randomly permuted, and $t$ values are calculated using the new sample groupings (Dudoit et al., 2000). Using 100 separate permutations of sample group identities, a control distribution for each replicate line was established from the $100 \times 4305$ (MAS version 4.0) or $100 \times 3989$ (MAS version 5.0) individual $t$ values. These control distributions were then used to determine thresholds at varying percentiles to filter the genes whose expression level changes exceeded that threshold. At each percentile, the threshold value used was the higher of the two values obtained (i.e., one from HAFT-1/ LAFT-1, one from HAFT-2/LAFT-2 data). The method of Eaves et al. (2002) was used to calculate the number of expected false positives. Lists of differentially-expressed genes (same direction in both replicate lines) identified by these two methods can be found in supplementary information at http://www2.uchsc.edu/pharm/faculty/tabakoff.suppl.asp.

Analysis of chromosomal localization and overlapping QTLs

The determination of chromosomal localization of differentially expressed known genes and comparison with the location of QTLs for AFT were performed using software developed by the Center for Computational Pharmacology at the University of Colorado Health Sciences Center (this software is available at http://inia.uchsc.edu). This software integrates the Affymetrix data, QTL data, and data from the The Jackson Laboratory (Bar Harbor, ME) Mouse Genome Information database. To determine the chromosomal localization of expressed sequence tags (ESTs) (DNA sequences) on the arrays, as well as sequence homology to known genes, and gene function, the batch query tool available at NetAffx Analysis Center (provided by Affymetrix, Inc.; www.affymetrix. com/analysis/index.affx) was used. If the information on the ESTs was not available from NetAffx, manual mapping was carried out, where possible, using LocusLink (www.ncibi.nlm.nih.gov/locuslink/) and the Mouse Genome Informatics database from The Jackson Laboratory (www.informatics.jax.org/). Once chromosomal localization of the ESTs was determined, it was manually compared with the QTLs for AFT.

After the identification of differentially expressed, QTL-localized transcripts through this analysis, the overall significance of the differential expression of these transcripts in HAFT-1 versus LAFT-1 and HAFT-2 versus LAFT-2 mice was determined with a two-way ANOVA [effect of line (HAFT vs LAFT) and effect of group (HAFT-1, LAFT-1, HAFT-2, LAFT-2)]. For this analysis, the raw data for the identified transcripts derived using MAS version 4.0 and MAS version 5.0 were used (see legend to Table 1).

\section{Quantitative real-time PCR}

Quantitative real-time PCR was performed using the Applied Biosystems International Prism 7700 Sequence Detection System. Sequence-specific TaqMan probes and primer sets, designed using Applied Biosystems International Prism 7700 sequence detection software (Primer Express; Applied Biosystems International), were used to carry out quantitative realtime PCR. Probe and primer sequences are: for NR1, forward primer: $5^{\prime}$ GGTGGCCGTGATGCTGTAC-3'; reverse primer, 5' -TCGCTGTTCAC- 
CTTAAATCGG-3'; probe: 5'-TGCTGGACCGCTTCAGTCCCTTTG-3'; for zinc finger protein 179 , forward primer: $5^{\prime}$-CTGCACTGCAGAAGACCTGTG-3'; reverse primer: $5^{\prime}$-TCCGGAGGCATTGATTCGTA-3'; probe: 5'-TGTGAGGGCAGAACGTCTGCTGTTG-3'. The TaqMan probes were purchased from PerkinElmer Life Sciences (Boston, MA) 5'-labeled with 6-carboxyfluorescein and 3'-labeled with 6-carboxytetramethylrhodamine. Thermal cycling conditions were as follows: reverse transcription was performed at $48^{\circ} \mathrm{C}$ for $30 \mathrm{~min}$, followed by the activation of AmpliTaq Gold at $95^{\circ} \mathrm{C}$ for $10 \mathrm{~min}$. Subsequently, 40 cycles of amplification were performed at $95^{\circ} \mathrm{C}$ for $15 \mathrm{sec}$ and $60^{\circ} \mathrm{C}$ for $1 \mathrm{~min}$.

The fluorescence data were expressed as normalized reporter signal ( Rn) or $\Delta \mathrm{Rn}$. Rn was calculated by dividing the amount of reporter signal by the amount of passive reference signal. $\Delta \mathrm{Rn}$ represents the amount of normalized reporter signal minus the amount of reporter signal before PCR. The detection threshold was set above the mean baseline fluorescence determined from the first 15 cycles. Amplification reactions in which the fluorescence intensity increased above the threshold were defined as positive. A standard curve for each template was generated using a serial dilution of the template (total RNA). Quantities of template in test samples were normalized to the corresponding $18 S$ rRNA.

Determination of transcription factor binding consensus sequences in differentially expressed genes

Dr. Razvan Lapadat, from the Center for Computational Pharmacology, University of Colorado Health Sciences Center, used information available from both the public domain and private databases to identify the transcription factor consensus binding sequence: (1) GenBank sequences, (2) LocusLink and RefSeq annotated entries, (3) UniGene clusters grouping all DNA and protein sequences belonging to one gene, and (4) Celera Genomics data (when no available genomic information was found in the public databases). This information was used to extract the 5 '-UTR sequence information for the analysis process. The transcription factor binding prediction is done using the TRANSFAC version 5.4 matrices (www.biobase.de). This database currently contains 487 binding matrices across all species. Composite regulatory element analysis was done using TransCompel version 3.0 (online version).

The $5^{\prime}$-UTR region was obtained by extracting the sequence data from the mouse section of the Celera Genomics database using the RefSeq identifiers. The start codons were found by using the GenBank RefSeq sequence information about the codon start position and generating 12 -mer splice tags that were matched against the genomic sequence. The 1 and $2 \mathrm{~kb}$ upstream sequences were retrieved and a matrix-based similarity search against the TRANSFAC matrices was performed using the pattern search for transcription factor binding sites (PATCH) program. The core and overall matrix similarity score cutoffs were set at 0.9 . The upstream sequences were searched against the TransCompel database using a maximum mismatch of 1 base pair, 30\% maximum distance variation compared with the original composite element (CE) binding sites, and a minimum composite score of 0.3 . The composite score is calculated as follows: composite score $=2 \times 10^{-m 1}+2 \times 10^{-m 2}+$ $\left[\left(10^{-g}\right) / 5\right]$, where $m 1$ and $m 2=$ number of mismatched nucleotides in promoter cores 1 and 2 and $g=$ (distance between sites in the known $\mathrm{CE})-($ distance in the found potential CE). Results shown are from the $2 \mathrm{~kb}$ upstream sequence search.

\section{Comparison of mouse and human QTLs}

Human-mouse chromosome homology maps (available at the National Center for Biotechnology Information website: www.ncbi.nlm.nih.gov/ Homology/) were searched to obtain the syntenic regions of the human genome corresponding to the mouse AFT/BEC2 QTLs. These syntenic regions of the human genome were then manually aligned with the QTLs for low alcohol response in humans (Schuckit et al., 2001). This procedure allows estimation of the overlap of the human QTL regions with the mouse QTLs for AFT/BEC2 in which we identified differentially expressed genes.

\section{Results}

\section{AFT measures}

As shown in Figure 1, HAFT mice of both replicate lines used for these experiments displayed greater AFT to the incoordinating
A

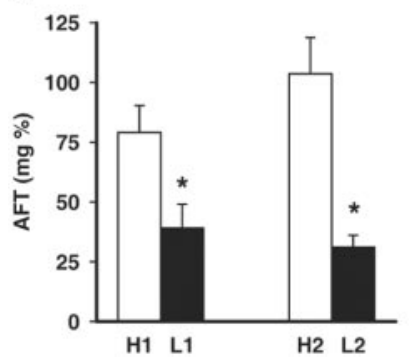

B

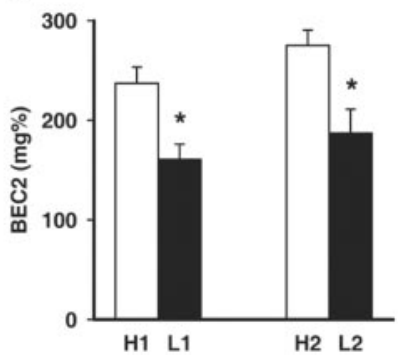

Figure 1. AFT and BEC2 in HAFT $(\mathrm{H})$ and LAFT (L) mice. HAFT and LAFT mice from the same generations of the replicate lines (1 and 2 ) that were used for the microarray study were tested for AFT to the incoordinating effect of ethanol $(A)$ and $B E C 2(B)$, as described previously (Erwin et al., 2000; Kirstein et al., 2002) (see Materials and Methods). ${ }^{*} p<0.05 ; t$ test $(n=$ 3-5/group)

effect of ethanol than their LAFT counterparts. HAFT mice also displayed higher levels of BEC2 than LAFT mice (see Materials and Methods).

\section{Gene expression differences in HAFT and LAFT mice}

The procedure described by Eaves et al. (2002) was initially used to identify differentially expressed transcripts. This method (" $t$ test noise distribution”; see Materials and Methods and Table 1) takes advantage of the availability of the replicate lines of HAFT and LAFT mice, assuming that the replicate lines will display similar differences in expression of genes that are associated with AFT (Crabbe et al., 1990, 1999). Data from the Affymetrix arrays were analyzed using MAS version 4.0 or MAS version 5.0, and the total number of transcripts that were analyzed, the number of genes and ESTs found to show statistically significant differences between lines at each threshold, as well as the expected percentage of false positives are shown in Table 1. Only genes/ESTs that displayed the same direction of differential expression in both replicate lines were considered. We chose to use a threshold of the 80th percentile for this array-wide comparison; i.e., we required that the value of $t$ in each comparison exceeded the 80th percentile of the control distribution. This threshold provides high sensitivity and therefore avoids exclusion of genes that warrant additional investigation (false negatives), at the expense of specificity.

Table 1 also shows the results of the permutation analysis, which has been suggested as a method for adjusting $p$ values obtained with multiple comparisons (Dudoit et al., 2000). This analysis was also performed on data obtained using MAS version 4.0 or MAS version 5.0. Again, only genes with the same direction of differential expression in both replicate lines of mice were considered further. In this analysis, a significance threshold was again chosen at the 80th percentile to avoid exclusion of possibly important genes.

It has been reported previously (Hoffmann et al., 2002) that the procedure used to normalize microarray data has a greater influence on the detection of differentially expressed genes than the statistical method used to determine differences. To determine the influence of our initial data analysis (MAS version $4.0 \mathrm{vs}$ MAS version 5.0), as compared with the statistical method used for setting thresholds for differential expression ( $t$ test noise distribution vs permutation), we compared the number of common differentially expressed transcripts detected when each single statistical method was used to analyze MAS version 4.0 and MAS version 5.0 data, or when both statistical methods were used to analyze the same data (i.e., derived from MAS version 4.0 or MAS 


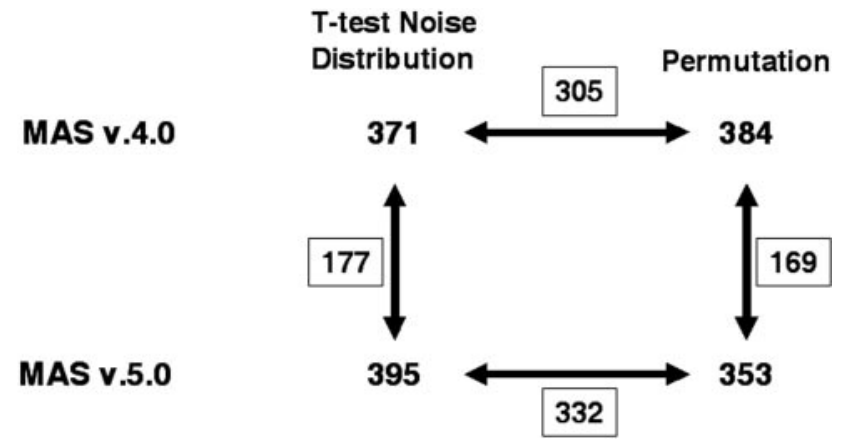

Figure 2. Effect of analysis procedures on detection of differentially expressed transcripts. The number of differentially expressed transcripts (differential expression in the same direction in both replicate lines) determined by the two statistical procedures ( $t$ test noise distribution or permutation, using the 80th percentile cutoff) when data are initially analyzed using MAS version 4.0 or MAS version 5.0, are indicated at the corners of the square. The common differentially expressed transcripts are indicated by the boxed numbers adjacent to the connecting lines. For example, when data analyzed initially with MAS version 4.0 were subjected to statistical analysis by the $t$ test noise distribution or the permutation procedure, 305 common transcripts were detected. There were 144 common differentially expressed transcripts regardless of the statistical analysis or MAS version used.

version 5.0). As shown in Figure 2, the initial normalization/ selection method (MAS version 4.0 or MAS version 5.0 ) had a greater influence on the number of common differentially expressed transcripts that were detected than the statistical method used. That is, there were fewer common genes (i.e., 169, 177) when the two normalization/selection methods were compared versus the number of common genes (i.e., 305, 332) that were evident when data obtained with a particular normalization/selection method were analyzed by two different statistical methods. This result supports the previous finding of the influence of the normalization procedure on the detection of differentially expressed genes (Hoffmann et al., 2002) and emphasizes the necessity either to use the same normalization procedure for all arrays in an experiment or to use more than one normalization/ selection method to detect transcripts that are found by all methods, and thus display the most robust changes.

We determined the chromosomal localization of the genes corresponding to the transcripts that were differentially expressed in both replicate lines. Comparison of these loci with the location of provisional QTLs previously identified for AFT and the highly genetically correlated trait, BEC2 (Kirstein et al., 2002), led to the list of genes shown in Table 2. Regardless of the initial data analysis software used (MAS version 4.0 or MAS version 5.0 ), and regardless of the statistical method used, six genes were identified as being differentially expressed between HAFT and LAFT mice and present in QTLs for AFT or BEC2. A two-way ANOVA revealed significant main effects of line (HAFT vs LAFT), with no significant line by group interaction (HAFT-1, LAFT-1, HAFT2, LAFT-2) (Table 2 legend). All but one of the differentially expressed genes localized within the QTLs were expressed at higher levels in HAFT than in LAFT mice. It may be noteworthy that there appears to be a cluster of differentially expressed genes located within one particular QTL (chromosome 11). There was also one differentially expressed EST (D11E17e) that was localized within the QTL on chromosome 11 (11:37), and was identified regardless of the initial data analysis or statistical method used. This EST was homologous to the Saccharomyces cerevisiae gene MUM2, which plays a role in DNA replication (Davis et al., 2001). Certain other genes that map to QTL regions were also identified as being differentially expressed at the 80th
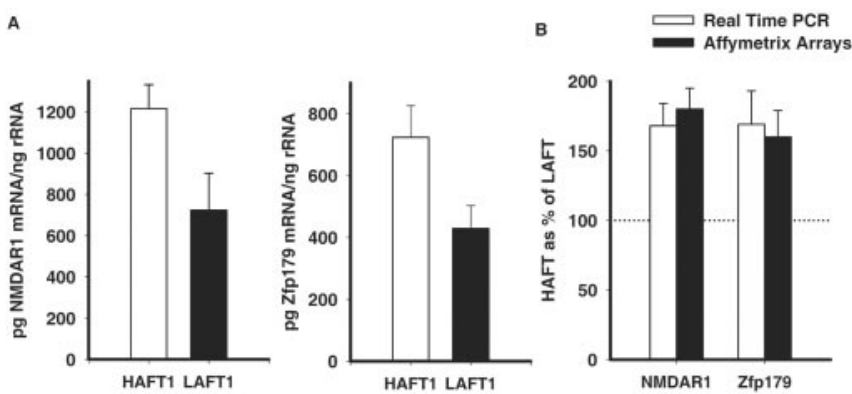

Figure 3. Verification of microarray data by real-time $P C R$. Real-time $P C R$ was performed using whole-brain total RNA from HAFT-1 and LAFT-1 mice (generation 24). The primer and probe sequences for NR 1 and zinc finger protein 179 are provided in Materials and Methods. $A$, Quantitation of NR1 and zinc finger protein 179 mRNA levels are based on a standard curve and normalized to 185 rRNA (Materials and Methods). B, The differential expression of NR1 and zinc finger protein 179 mRNA between HAFT and LAFT mice was quantitatively similar when measured by microarrays or real-time $P C R$.

Table 3. Putative promoters for differentially expressed genes

\begin{tabular}{lllll}
\hline C/EBP- $\alpha$ & $\begin{array}{l}\text { cAMP response element- } \\
\text { binding protein }\end{array}$ & Myosin D & AP-1 & $\begin{array}{l}\text { Nuclear factor } \\
\kappa \mathrm{B}\end{array}$ \\
\hline $\begin{array}{l}\text { Tcebll } \\
\text { Zfp179 }\end{array}$ & Tcebll & & Tcebll & \\
Prdx5 & Prdx5 & Zfp179 & & \\
Efnb3 & Efnb3 & & & Efnb3 \\
Grin1 & Grin1 & Efnb3 & & \\
\hline
\end{tabular}

percentile level by both statistical analyses, but these statistical differences were evident only with either one normalization/ selection procedure or the other (MAS version 4.0 or MAS version 5.0) (Table 1).

Real-time PCR was used to verify the differential expression of some of the genes identified by microarray and QTL analysis. Figure 3 shows that not only was the differential expression verified by real-time PCR, but also that the magnitude of differences in expression was similar for both methods. Our microarray data showed excellent reproducibility among samples [data available as supplemental information at www.jneurosci.org (Fig.1)], and similar results have been reported by others (Hoffman et al., 2003). Given this reproducibility, and the nearly identical results obtained by real-time PCR compared with the microarray data, we conclude that the reliability of the data obtained from microarrays is at least equal to that obtained by real-time PCR for detecting statistically significant differences in transcript expression. Data showing that protein levels parallel mRNA levels for some of the differentially expressed transcripts are also provided in the supplementary information [available at www.jneurosci.org (Fig. 2)]

One explanation for the differential expression of the genes between HAFT and LAFT mice is that a polymorphism in the $5^{\prime}$ regulatory region of certain genes underlies the QTL for AFT. As an initial step in exploring this possibility, we identified consensus sequences for transcription factor binding sites in the differentially expressed genes. As shown in Table 3, all of the genes identified using both initial analysis methods and both statistical techniques, and for which information was available (see Materials and Methods), had the consensus sequence for the CCAAT/ enhancer binding protein $(\mathrm{C} / \mathrm{EBP} \alpha)$, which is a transcription factor that has both constitutive and cAMP-inducible activities (Wilson et al., 2001; Wilson and Roesler, 2002). In addition, each of the differentially expressed genes had one or more other transcription factor binding sites. 


\section{Discussion}

This study describes a novel method for identifying candidate genes associated with a complex behavior. The method combines the use of lines of animals that have been selectively bred for differences in behavior, i.e., AFT to ethanol, with DNA microarray analysis, and QTL analysis. In the present study, we used a high sensitivity threshold to initially estimate differential brain gene expression between selected lines of HAFT and LAFT mice, resulting in the identification of $>300$ genes that displayed differential expression and that showed the same direction of difference in both replicate lines. Because of the high sensitivity but low specificity of the initial screen, there are likely to be a number of false positives among the initially identified, differentially expressed genes. Specificity was subsequently greatly enhanced by adding filters that limited the genes to those wherein: (1) differences had to be statistically significant and be in the same direction in both of the selected lines of mice, (2) the differentially expressed genes had to be localized within QTLs associated with AFT, and (3) differences had to be statistically significant regardless of the initial microarray analysis procedure or statistical method used to detect differences. These procedures allow us to more definitively identify a set of genes that can be considered with some confidence to be important for AFT to ethanol.

There are certain caveats that must be considered with respect to the present identification of genes associated with the phenotype of AFT. For instance, the differentially expressed genes that we have identified are localized within some, but not all, of the provisional QTLs that have been determined for AFT (Kirstein et al., 2002). The current method clearly will not identify genes that lie within QTLs, and that differ in their coding regions, such that the function of the gene product may be altered. It is also important to note that although the HAFT and LAFT lines also differ in the development of tolerance to the incoordinating effect of ethanol as measured on a rotarod apparatus (Deitrich et al., 2000; Rustay et al., 2001), they do not differ in tolerance to certain other effects of ethanol (e.g., hypnotic effect, change in body temperature; Deitrich et al., 2000; Erwin et al., 2000). Therefore, the association of the identified genes cannot at this time be generalized to acute tolerance to all physiological/pharmacological effects of ethanol.

However, there are reasons to be confident that the genes that have been identified are associated with AFT to the incoordinating actions of ethanol. As mentioned, many of the differentially expressed genes localized within QTLs were identified regardless of the method for analysis of the array data or the statistical method used to determine differential expression. The restriction that genes had to be differentially expressed in the same direction in both replicate lines of selected HAFT and LAFT animals also provides confidence in the results, because the replicate lines represent completely independent selections for the same phenotype; i.e., different populations of heterogeneous stock mice were used to start the two selective breeding experiments. The use of replicate lines of selected animals has been recommended because the observation of the same biochemical difference in both lines provides more confidence that the difference is associated with the selected phenotype (Crabbe et al., 1990). It is also important to note that the QTL analysis was performed using the identical behavioral test that was used for selection (Erwin and Deitrich, 1996; Kirstein et al., 2002). Therefore, there is a high degree of probability that the differentially expressed genes that are localized within the QTLs are involved in AFT to the incoordinating effect of ethanol.
Some of the differentially expressed genes that are localized within the QTLs for AFT can be readily related to the measured behavior and can be positioned within a signal transduction pathway that includes elements previously associated with neuroadaptation. A more detailed discussion of this pathway can be found in the supplementary information at http://www2.uchsc. edu/pharm/faculty/tabakoff/suppl.asp. Briefly, the glutamate receptor $\delta 2(\mathrm{GluR} \delta 2)$ protein, which is expressed exclusively in the cerebellum, is involved in motor coordination (Araki et al., 1993; Kashiwabuchi et al., 1995; Zuo et al., 1997; Lalouette et al., 2001), but it is also physically associated with kinases and phosphatases that can increase tyrosine phosphorylation of the NMDA receptor (Roche et al., 1999; Hironaka et al., 2000; Miyagi et al., 2002). Ephrin B3, an ephrin ligand, activates ephrin receptors that are tyrosine kinases, and activation of the ephrin system also increases NMDA receptor phosphorylation (Kullander and Klein, 2002; Murai and Pasquale, 2002) and affects clustering of the NR1 subunit of the NMDA receptor (Dalva et al., 2000). Tyrosine phosphorylation of the NMDA receptor by the nonreceptor tyrosine kinase Fyn has been reported previously to be a key factor in AFT to the inhibitory effect of ethanol on the NMDA receptor (Miyakawa et al., 1997). Peroxidredoxin is an antioxidant protein that may interfere with the downstream function of the NMDA receptor (Chae et al., 1994; Marin et al., 1992; Cambonie et al., 2000). Thus, the combination of lower levels of GluR $\delta 2$, Ephrin $\mathrm{B} 3$, and the NMDA receptor subunit NR1, as well as the higher level of peroxiredoxin, provides a picture of downregulated NMDA receptor function in the brains of LAFT mice, and particularly provides a basis for concluding that tyrosine phosphorylation of the receptor, which has been implicated in AFT to ethanol, would be impaired in LAFT mice. Given the exclusive localization of GluR $\delta 2$ in cerebellar Purkinje cells, and the evidence for functional NMDA receptors in these cells (Thompson et al., 2000; Misra et al., 2000; Miyagi et al., 2002), the data suggest that impaired NMDA receptor phosphorylation/function in the cerebellum or other brain regions of LAFT mice may be a contributing factor in the reduced AFT to the incoordinating effect of ethanol in these mice.

The other genes that showed consistent differences in expression, and that were localized within QTLs for AFT, were the zinc finger protein 179 and a transcription elongation factor. Both of these genes control transcriptional events (Sowden et al., 1995; Reines et al., 1999), and the zinc finger protein 179 is member of the RING finger (zinc binding domain) family, that can be induced rapidly by activation of NMDA receptors (Zhao et al., 1998; Ohkawa et al., 2001). The lower expression of these genes in the brains of LAFT mice is likely to influence the expression of other specific genes that may, in a secondary manner, contribute to AFT to ethanol. A schematic illustration of pathways linking the differentially expressed genes is shown in the supplementary information at http://www2.uchsc.edu/pharm/faculty/tabakoff/ suppl.asp.

We analyzed the differential expression of genes in whole brains of HAFT and LAFT mice because there was no $a$ priori basis for choosing a brain area to be associated with AFT. The selective expression of certain genes of interest (e.g., GluR $\delta 2$ ) in the cerebellum provides some post hoc evidence that a particular brain area may be functionally important to the development of AFT to the incoordinating effect of ethanol.

The differentially expressed genes within QTLs for AFT could reflect polymorphisms in regulatory regions of the genes that affect transcription factor binding. As an initial exploration of this possibility, we have used bioinformatic techniques to assess 
putative transcription factor binding sites associated with the differentially expressed genes. Additional work will be needed to unravel the possible differences in the sequence or combinations of sequences that may contribute to the differential expression of the identified genes in HAFT and LAFT mice.

Clinical studies by Schuckit and Smith (2000) have indicated that individuals with a positive family history of alcohol dependence (FHP) show blunted physiological, behavioral (including incoordination), and subjective responses to alcohol ("low response to alcohol") compared with those with a negative family history of alcohol dependence. It has been suggested that this blunted response may reflect greater levels of AFT in the FHP individuals (Newlin and Thomson, 1990). The low alcohol responders (primarily FHP) have been found to have a significantly greater risk of becoming alcohol-dependent (Schuckit and Smith, 2000). Schuckit et al. (2001) recently identified four chromosomal regions in humans that were correlated with a low level of response to alcohol. We compared the identified human chromosomal regions to the QTL regions for AFT in mice to determine whether the differentially expressed genes that we had identified might lie within regions of the human genome associated with AFT/low response to ethanol in humans. By examining the syntenic regions of mouse and human genomes we were able to determine that two mouse QTLs for AFT (chromosomal locations: 11:29-43 and 1:81.1-89.2) were syntenic with regions of the human genome that had been identified by Schuckit et al. (2001) as QTLs for low response to ethanol. Of the four genes located in the mouse QTLs, two (transcription elongation factor and peroxiredoxin 5) were located within or near the human QTL regions. Whether the identified mouse genes within these QTLs also show different expression between humans with a high and low response to ethanol remains to be determined.

\section{References}

Affymetrix (2001) Statistical algorithms reference guide. Santa Clara, CA: Affymetrix, Inc.

Aitman TJ, Glazier AM, Wallace CA, Cooper LD, Norsworthy PJ, Wahid FN, Al-Majali KM, Trembling PM, Mann CJ, Shoulders CC, Graf D, St Lezin E, Kurtz TW, Kren V, Pravenec M, Ibrahimi A, Abumrad NA, Stanton LW, Scott J (1999) Identification of Cd36 (Fat) as an insulin-resistance gene causing defective fatty acid and glucose metabolism in hypertensive rats. Nat Genet 21:76-83.

American Psychiatric Association (1994) Diagnostic and statistical manual of mental disorders. Washington, DC: American Psychiatric Association.

Araki K, Meguro H, Kushiya E, Takayama C, Inoue Y, Mishina M (1993) Selective expression of the glutamate receptor channel delta 2 subunit in cerebellar Purkinje cells. Biochem Biophys Res Commun 197:1267-1276.

Belknap JK, Mitchell SR, O’Toole LA, Helms ML, Crabbe JC (1996) Type I and type II error rates for quantitative trait loci (QTL) mapping studies using recombinant inbred mouse strains. Behav Genet 26:149-160.

Cambonie G, Laplanche L, Kamenka JM, Barbanel G (2000) N-methyl-Daspartate but not glutamate induces the release of hydroxyl radicals in the neonatal rat: modulation by group I metabotropic glutamate receptors. J Neurosci Res 62:84-90.

Chae HZ, Robison K, Poole LB, Church G, Storz G, Rhee SG (1994) Cloning and sequencing of thiol-specific antioxidant from mammalian brain: alkyl hydroperoxide reductase and thiol-specific antioxidant define a large family of antioxidant enzymes. Proc Natl Acad Sci USA 91:7017-7021.

Crabbe JC, Phillips TJ, Kosobud A, Belknap JK (1990) Estimation of genetic correlation: interpretation of experiments using selectively bred and inbred animals. Alcohol Clin Exp Res 14:141-151.

Crabbe JC, Phillips TJ, Buck KJ, Cunningham CL, Belknap JK (1999) Identifying genes for alcohol and drug sensitivity: recent progress and future directions. Trends Neurosci 22:173-179.

Dalva MB, Takasu MA, Lin MZ, Shamah SM, Hu L, Gale NW, Greenberg ME
(2000) EphB receptors interact with NMDA receptors and regulate excitatory synapse formation. Cell 103:945-956.

Davis L, Barbera M, McDonnell A, McIntyre K, Sternglanz R, Jin Q, Loidl J, Engebrecht J (2001) The Saccharomyces cerevisiae MUM2 gene interacts with the DNA replication machinery and is required for meiotic levels of double strand breaks. Genetics 157:1179-1189.

Deitrich RA, Bludeau P, Erwin VG (2000) Phenotypic and genotypic relationships between ethanol tolerance and sensitivity in mice selectively bred for initial sensitivity to ethanol (SS and LS) or development of acute tolerance (HAFT and LAFT). Alcohol Clin Exp Res 24:595-604.

Dudoit S, Yang YH, Callow MJ, Speed TP (2000) Statistical methods for identifying differentially expressed genes in replicate cDNA microarray experiments. Stanford, CA: Stanford University School of Medicine. http://www.stat.berkeley.edu/users/terry/zarray/techreport/578.pdf

Eaves IA, Wicker LS, Ghandour G, Lyons PA, Peterson LB, Todd JA, Glynne RJ (2002) Combining mouse congenic strains and microarray gene expression analyses to study a complex trait: the NOD model of type 1 diabetes. Genome Res 12:232-243.

Erwin VG, Deitrich RA (1996) Genetic selection and characterization ofmouse lines for acute functional tolerance to ethanol. J Pharmacol Exp Ther 279:1310-1317.

Erwin VG, Gehle VM, Deitrich RA (2000) Selectively bred lines of mice show response and drug specificity for genetic regulation of acute functional tolerance to ethanol and pentobarbital. J Pharmacol Exp Ther 293:188-195.

Falconer DS, Mackay TFC (1996) Introduction to quantitative genetics. Essex, UK: Longman.

Hironaka K, Umemori H, Tezuka T, Mishina M, Yamamoto T (2000) The protein-tyrosine phosphatase PTPMEG interacts with glutamate receptor $\delta 2$ and $\epsilon$ subunits. J Biol Chem 275:16167-16173.

Hoffmann R, Seidl T, Dugas M (2002) Profound effect of normalization on detection of differentially expressed genes in oligonucleotide microarray data analysis. Genome Biol 3:1-11.

Hoffman PL, Miles M, Edenberg HJ, Sommer W, Tabakoff B, Wehner JM, Lewohl J (2003) Gene expression in brain: a window on ethanol dependence, neuroadaptation, and preference. Alcohol Clin Exp Res 27:155-168.

Karp CL, Grupe A, Schadt E, Ewart SL, Keane-Moore M, Cuomo PJ, Kohl J, Wahl L, Kuperman D, Germer S, Aud D, Peltz G, Wills-Karp M (2000) Identification of complement factor 5 as a susceptibility locus for experimental allergic asthma. Nat Immunol 1:221-226.

Kashiwabuchi N, Ikeda K, Araki K, Hirano T, Shibuki K, Takayama C, Inoue Y, Kutsuwada T, Yagi T, Kang Y, et al (1995) Impairment of motor coordination, Purkinje cell synapse formation, and cerebellar long-term depression in GluR $\delta 2$ mutant mice. Cell 81:245-252.

Kirstein SL, Davidson KL, Ehringer MA, Sikela JM, Erwin VG, Tabakoff B (2002) Quantitative trait loci affecting initial sensitivity and acute functional tolerance to ethanol-induced ataxia and brain cAMP signaling in BXD recombinant inbred mice. J Pharmacol Exp Ther 302:1238-1245.

Kullander K, Klein R (2002) Mechanisms and functions of eph and ephrin signalling. Nat Rev Mol Cell Biol 3:475-486.

Lalouette A, Lohof A, Sotelo C, Guenet J, Mariani J (2001) Neurobiological effects of a null mutation depend on genetic context: comparison between two hotfoot alleles of the $\delta$-2 ionotropic glutamate receptor. Neuroscience 105:443-455.

Lander E, Kruglyak L (1995) Genetic dissection of complex traits: guidelines for interpreting and reporting linkage results. Nat Genet 11:241-247.

Marin P, Lafon-Cazal M, Bockaert J (1992) A nitric oxide synthase activity selectively stimulated by NMDA receptors depends on protein kinase C activation in mouse striatal neurons. Eur J Neurosci 4:425-432.

Misra C, Brickley SG, Wyllie DJ, Cull-Candy SG (2000) Slow deactivation kinetics of NMDA receptors containing NR1 and NR2D subunits in rat cerebellar Purkinje cells. J Physiol (Lond) 525:299-305.

Miyagi Y, Yamashita T, Fukaya M, Sonoda T, Okuno T, Yamada K, Watanabe M, Nagashima Y, Aoki I, Okuda K, Mishina M, Kawamoto S (2002) Delphilin: a novel PDZ and formin homology domain-containing protein that synaptically colocalizes and interacts with glutamate receptor $\delta 2$ subunit. J Neurosci 22:803-814.

Miyakawa T, Yagi T, Kitazawa H, Yasuda M, Kawai N, Tsuboi K, Niki H 
(1997) Fyn-kinase as a determinant of ethanol sensitivity: relation to NMDA-receptor function. Science 278:698-701.

Murai KK, Pasquale EB (2002) Can Eph receptors stimulate the mind? Neuron 33:159-162.

Newlin DB, Thomson JB (1990) Alcohol challenge with sons of alcoholics: a critical review and analysis. Psychol Bull 108:383-402.

Ohkawa N, Kokura K, Matsu-Ura T, Obinata T, Konishi Y, Tamura TA (2001) Molecular cloning and characterization of neural activity-related RING finger protein (NARF): a new member of the RBCC family is a candidate for the partner of myosin V. J Neurochem 78:75-87.

Reines D, Conaway RC, Conaway JW (1999) Mechanism and regulation of transcriptional elongation by RNA polymerase II. Curr Opin Cell Biol 11:342-346.

Roche KW, Ly CD, Petralia RS, Wang YX, McGee AW, Bredt DS, Wenthold RJ (1999) Postsynaptic density-93 interacts with the $\delta 2$ glutamate receptor subunit at parallel fiber synapses. J Neurosci 19:3926-3934.

Rustay NR, Boehm II SL, Schafer GL, Browman KE, Erwin VG, Crabbe JC (2001) Sensitivity and tolerance to ethanol-induced incoordination and hypothermia in HAFT and LAFT mice. Pharmacol Biochem Behav 70:167-174.

Schuckit MA, Smith TL (2000) The relationships of a family history of alcohol dependence, a low level of response to alcohol and six domains of lifefunctioning to the development of alcohol use disorders. J Stud Alcohol 61:827-835.

Schuckit MA, Edenberg HJ, Kalmijn J, Flury L, Smith TL, Reich T, Bierut L,
Goate A, Foroud T (2001) A genome-wide search for genes that relate to a low level of response to alcohol. Alcohol Clin Exp Res 25:323-329.

Sowden J, Morrison K, Schofield J, Putt W, Edwards Y (1995) A novelcDNA with homology to an RNA polymerase II elongation factor maps to human chromosome 5q31 (TCEB1L) and to mouse chromosome 11 (Tceb11). Genomics 29:145-151.

Thompson CL, Drewery DL, Atkins HD, Stephenson FA, Chazot PL (2000) Immunohistochemical localization of $N$-methyl-D-aspartate receptor NR1, NR2A, NR2B and NR2C/D subunits in the adult mammalian cerebellum. Neurosci Lett 283:85-88.

Wilson HL, Roesler WJ (2002) CCAAT/enhancer binding proteins: do they possess intrinsic cAMP-inducible activity? Mol Cell Endocrinol 188:15-20.

Wilson HL, McFie PJ, Roesler WJ (2001) Characterization of domains in $\mathrm{C} / \mathrm{EBP} \alpha$ that mediate its constitutive and cAMP-inducible activities. Mol Cell Endocrinol 181:27-34.

World Health Organization (1993) The ICD-10 classification of mental and behavioral disorders, diagnostic criteria for research. Geneva: World Health Organization.

Zhao Q, Chen KS, Bejjani BA, Lupski JR (1998) Cloning, genomic structure, and expression of mouse ring finger protein gene Znf179. Genomics 49:394-400.

Zuo J, De Jager PL, Takahashi KA, Jiang W, Linden DJ, Heintz N (1997) Neurodegeneration in Lurcher mice caused by mutation in $\delta 2$ glutamate receptor gene. Nature 388:769-773. 